\title{
\begin{tabular}{l|l} 
POLITIQUES \& & Politiques et management public
\end{tabular}

\section{Le conseil en stratégies et projets urbains : un marché atypique}

The urban planning and strategy consulting: an atypical market

\section{Rachel Linossier}

\section{(2) OpenEdition \\ Journals}

Electronic version

URL: http://journals.openedition.org/pmp/4733

ISSN: 2119-4831

\section{Publisher}

Institut de Management Public (IDPM)

\section{Printed version}

Date of publication: 15 January 2012

Number of pages: $57-78$

ISBN: $978-2-7430-1437-7$

ISSN: 0758-1726

\section{Electronic reference}

Rachel Linossier, «Le conseil en stratégies et projets urbains : un marché atypique », Politiques et management public [En ligne], Vol 29/1 | 2012, mis en ligne le 20 juin 2014, consulté le 19 avril 2019 URL : http://journals.openedition.org/pmp/4733 


\title{
Le conseil en stratégies et projets urbains : 04 un marché atypique
}

\author{
Rachel Linossier \\ MCF IUT 2, Université Grenoble 2, \\ PACTE UMR 5194 (CNRS, IEPG, UJF, UPMF), 38041 Grenoble
}

\section{Résumé}

Les trajectoires professionnelles des consultants et l'activité des cabinets de conseils en stratégies et projets urbains mettent en évidence le caractère atypique de ce segment du marché du conseil en France. Son fonctionnement est pour une large part hérité de la période des Trente Glorieuses. Il présente des contours flous, il est très segmenté et fragile financièrement. II est encore largement dominé par le secteur public (État, collectivités locales, agences d'urbanisme), ce qui induit des distorsions de concurrence. Pour s'affranchir des conditions inégales d'accès à la commande, les consultants privés développent des niches de marché, activent leurs réseaux socioprofessionnels, militants ou politiques et jouent la carte de la pluriactivité, en valorisant leur expérience dans le champ de l'urbanisme et de l'aménagement.

(c) 2012 IDMP/Lavoisier SAS. Tous droits réservés

Mots clés : Urbanisme, marché, consultant, cabinet de conseil, carrière professionnelle

\section{Abstract}

The urban planning and strategy consulting: an atypical market. Professional careers of the consultants and the activity of the urban planning and strategy consulting firms show how atypical is this segment of the consulting market in France. The way it works is inherited from the Glorious Thirty. It is a hardly defined, a highly segmented and a financially fragile market. It is still largely dominated by the public sector (State, local authorities, urban planning agencies) and it is not fully open to competition. The private consultants carve out market niches, they activate social, professional or political networks and play the multi-activity card to overcome the disparities in access to demand.

(c) 2012 IDMP/Lavoisier SAS. Tous droits réservés

Keywords: Urban planning, market, consultant, consulting firm, professional career

*Auteur correspondant : rachel.linossier@wanadoo.fr

doi:10.3166/pmp.29.57-78 @ 2012 IDMP/Lavoisier SAS. Tous droits réservés 


\section{Introduction}

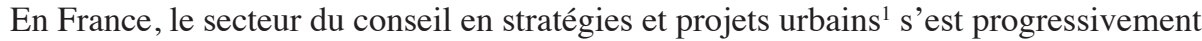
structuré depuis les années 1950. Il est assez restreint, comparativement à d'autres champs du conseil comme le conseil en management d'entreprises, le conseil en gestion de ressources humaines, le conseil en développement informatique ou le domaine des études de marché et d'opinion (Henry, 1992 ; Abiker, 1996). Il constitue un secteur d'activités à part et un marché atypique, car ses caractéristiques et son fonctionnement ne correspondent pas aux canons classiques de l'échange économique, notamment en termes de concurrence, de pluralité de l'offre et de la demande ou d'accès à la commande. Ce champ professionnel est en effet caractérisé par une grande atomicité et une forte instabilité structurelles, avec beaucoup de créations et de disparitions, de fusions ou de rachats, qui empêchent toute tentative d'identification de la totalité des acteurs de ce marché (Reggazzola, 1988 ; Dubus, 1999 ; Roux, 2003). Son fonctionnement présente également d'importants phénomènes de distorsion de la concurrence, résultant en grande partie de l'omniprésence du secteur public, tant du côté de la demande que du côté de l'offre de conseil, qui conduisent les consultants et leurs cabinets à déployer des stratégies commerciales fondées sur l'activation de réseaux et la diversification de leur expérience professionnelle.

Ce constat procède d'une démarche d'analyse non exhaustive, appuyée sur l'étude qualitative des trajectoires professionnelles de plusieurs consultants en stratégies et projets urbains, visant à mettre en lumière les logiques de culture professionnelle, de partage et de diffusion des modèles de production de la ville qui caractérisent les parcours professionnels dans ce champ d'activités (Cf. Annexe méthodologique). La notion de « carrière », empruntée à la sociologie interactionniste (Becker, 1985), permet de prendre la dimension temporelle en compte dans l'analyse. Elle propose un modèle séquentiel différenciant les étapes successives du parcours personnel de chaque consultant, les phases de changement et les passages d'une position professionnelle à une autre, facilitant la compréhension de la genèse et de l'évolution des comportements propres à leur groupe, en lien avec les évolutions du contexte. Deux étapes importantes dans les carrières étudiées éclairent ainsi le fonctionnement atypique de ce marché : l'entrée de chaque consultant dans le champ professionnel de l'urbanisme et de l'aménagement, et la reconnaissance de son appartenance au groupe professionnel. La première renvoie au cursus de formation initiale et à la première expérience dans le champ, qui déterminent l'engagement professionnel et l'adoption par le consultant des valeurs et modèles de pensée de la profession. La seconde renvoie à l'existence d'une culture professionnelle partagée par l'ensemble des consultants (et par leurs donneurs d'ordre), ainsi qu'aux phénomènes de réseaux, d'échanges et de diffusion des idées, qui favorisent un accès privilégié de certains d'entre eux à la commande.

\footnotetext{
${ }^{1}$ Cette dénomination, volontairement floue et globalisante, est spontanément utilisée par plusieurs consultants enquêtés pour identifier leur secteur d'activités. Elle renvoie à un large éventail de prestations : conseils stratégiques ou techniques, études, expertises, audits, recherches, diagnostics, cahiers de préconisations ou des charges, assistance à maîtrise d'ouvrage ou à maîtrise d'œuvre (AMO), etc. Les domaines d'activités couverts sont également très divers : stratégie, prospective, planification urbaine et territoriale, développement urbain, économique et territorial, programmation urbaine, urbanisme opérationnel et projet urbain, politiques urbaines, etc.
} 
Cette approche par les carrières permet également de considérer l'évolution et les positionnements respectifs des organisations du champ étudié : les cabinets de consultants, les bureaux d'études $(\mathrm{BE})^{2}$ et les autres organismes publics ou assimilés intervenant dans ce champ d'activités du côté de l'offre (agences d'urbanisme, établissements de formation, laboratoires de recherche, etc.), mais aussi les services de l'État, les collectivités locales et certains de leurs organismes « satellites » (agences d'urbanisme, sociétés d'économie mixte) du côté de la demande. Elle permet ainsi d'envisager les modalités de la structuration de ce champ sous la forme d'un marché, en identifiant les caractéristiques intrinsèques de la demande et de l'offre, ainsi que les modalités de leur mise en relation.

\section{Une demande monopolisée par le secteur public}

Les spécificités structurelles de la demande de conseil en stratégies et projets urbains ne peuvent se comprendre qu'au regard du mode de constitution historique de ce secteur d'activités.

\subsection{L'histoire du marché des études urbaines en France}

Dans les 1960, de grands bureaux d'études (BE) pluridisciplinaires ont été chargés de préparer les Plans de Modernisation et d'Équipement pour le compte du Service Régional et Urbain du Commissariat Général au Plan (CGP), de produire des modèles économétriques d'urbanisation pour le compte de la Datar ${ }^{3}$ (dans le cadre de la politique des Métropoles d'équilibre puis des Villes nouvelles notamment) et de préparer les documents de planification urbaine pour le compte des services déconcentrés de l'État (Missions régionales d'aménagement, Oream et Gep des $\mathrm{DDE}^{4}$ ).

De statut privé, ils avaient des rattachements institutionnels divers et employaient chacun plusieurs dizaines de personnes. Certains étaient des filiales de groupes financiers privés comme Indosuez et Paribas $\left(\mathrm{Sema}^{5}\right.$, Métra International - Otam, Otu, Oth $\left.{ }^{6}\right)$ ou à capitaux publics comme la Caisse des Dépôts et Consignations (Sedes, Cerau, Beture) ${ }^{7}$. D'autres étaient liés à des organismes administratifs publics comme le CGP et le ministère

\footnotetext{
${ }^{2}$ La différence entre les deux appellations tient essentiellement à l'effet de mode - on parle plutôt de bureaux d'études dans les années 1960 et 1970 et de cabinets de conseil ou de consultants à partir des années 1980 -, mais aussi à la distinction entre des structures dominées par les approches techniques et l'ingénierie, travaillant principalement pour le compte de l'État, et des organismes inscrits dans les démarches gestionnaires et stratégiques, travaillant plutôt pour des collectivités locales et d'autres acteurs institutionnels locaux.

${ }^{3}$ Délégation à l'aménagement du territoire et à l'action régionale.

${ }^{4}$ Organismes régionaux d'Études des Aires métropolitaines, Groupes d'Études et de Programmation des Directions départementales de l'Équipement.

${ }^{5}$ Société d'économie et de mathématiques appliquées.

${ }^{6}$ Omnium technique de l'aménagement, Omnium technique des études urbaines et Omnium technique de l'habitat.

${ }^{7}$ Le Centre d'études et de recherches en aménagement urbain (Cerau) est issu de la fusion des départements « études urbaines » de la Société d'études pour le développement économique et social (Sedes), du Crédoc et de la Société centrale d'équipement du territoire (Scet). Il est absorbé par le Bureau d'études pour l'urbanisme et l'équipement (Beture) en 1971.
} 
des Finances $\left(\right.$ Bipe $\left.^{8}, \mathrm{Crédoc}^{9}\right)$ ou encore structurés sous forme de coopératives et autres sociétés anonymes privées (Béru, Sacec, Area). Ils ont bénéficié de la manne de crédits d'études étatiques de la politique d'aménagement du territoire et sont à l'origine de la constitution d'un «marché des études $»^{10}$ en France (Bardet, 2005). En répondant à cet important volume de commandes publiques - auxquelles ils accédaient très facilement, faisant littéralement « leur marché » auprès du Ministère de l'Équipement ou du CGP et contribuant bien souvent à la définition même des commandes - (Reggazzola, 1988), ils ont participé au «pouvoir d'expertise » de l'État sur les questions urbaines (Claude, 2006).

Leurs personnels étaient des ingénieurs fonctionnaires détachés ou en disponibilité du ministère de l'Équipement et des chargés d'études ou de recherche contractuels issus de cursus universitaires. Ils ont été porteurs d'un profond renouvellement des approches de l'aménagement urbain. Ils ont concouru à la mise en place de « combinaisons de compétences » (Claude, 2006, p. 210), ouvrant le champ des politiques urbaines à de nouvelles disciplines : économie, sociologie, géographie, science politique, droit, psychologie, sciences de l'ingénieur, etc. Ils ont aussi participé à la diffusion d'une méthodologie de l'action publique reposant sur de nouveaux référentiels empruntés au thème de la réforme, comme le développement économique, la gestion stratégique et la démarche de projet, qui connaîtront leur essor avec la Décentralisation (Berrebi-Hoffmann, Grémion, 2009). Leur capacité d'influence sur l'évolution des conceptions et des pratiques de l'urbanisme a été d'autant plus forte, qu'ils monopolisaient la production d'études en amont de la décision et s'inséraient dans un système d'expertise technocratique reposant sur les relations croisées entre la sphère des hauts fonctionnaires et celle des $\mathrm{BE}$. Cette porosité a notamment permis de «faire bénéficier l'administration du savoir-faire des bureaux d'études » (Claude, 2006, p. 174) et de diffuser de nouveaux référentiels et modèles de pensée auprès des acteurs de la ville (Abiker, 1996).

La carrière de plusieurs consultants en stratégies et projets urbains a commencé au sein de ces BE, dont la plupart ont disparu au cours des années 1970, terrassés par le tarissement des crédits d'études lors de l'arrêt des grandes politiques d'aménagement du territoire et d'urbanisation de l'État (Cf. Tableau 1, Annexe méthodologique). Les BE ont en effet largement essaimé dans les agences d'urbanisme d'agglomération, les collectivités locales, les instituts de formation aux métiers de l'urbanisme et les cabinets de consultants, qui ont pris le relais du pouvoir d'expertise de l'État. Au Cerau, certains chargés d'études ont poursuivi momentanément leurs activités au Beture ou à la Sacec, d'autres ont rejoint l'université ${ }^{11}$ ou réintégré leur administration d'origine. Le Béru est par exemple à l'origine de la création de la première agence d'urbanisme française ${ }^{12}$, puis a échappé à la tourmente en changeant de statut à travers la création du cabinet privé Codra. Celui-ci absorbe alors d'anciens membres de l'Area, dont J.-M. Roux, tandis que H. Huntzinger et un autre salarié de la Sacec s'associent pour créer le cabinet Tetra... Ces BE apparaissent ainsi comme des

\footnotetext{
${ }^{8}$ Bureau d'information et de prévisions économiques.

${ }^{9}$ Centre de recherches et de documentation sur la consommation.

${ }^{10}$ Expression empruntée à Weill G., Le problème des études urbaines au seuil du Ve Plan, Journées d'études de Fontainebleau, 6-7 avril 1965 (Claude, 2006).

${ }^{11}$ R. Prud'homme, universitaire et conseiller Cerau /Beture à temps partiel, devient professeur à I'Institut d'Urbanisme de Paris (Université Paris 12), où il fonde L'CEIL.

12 Société rouennaise d'études urbaines (Soretur), société civile d'études créée sous l'égide de la Scet et de la CDC en 1963 (Prévot, 2007).
} 
« incubateurs de carrières $»^{13}$, véritables ateliers de transformation d'ingénieurs, géographes, économistes et autres sociologues en concepteurs, praticiens et experts de l'aménagement et de l'urbanisme, qui accompagnent la modernisation de l'administration territoriale en fournissant des réponses aux nouveaux besoins de l'action publique locale.

\subsection{Un renouveau de la demande émanant des collectivités locales}

Depuis les premières lois de décentralisation au début des années 1980, la demande de conseil en stratégies et projets urbains s'est développée, mais elle émane toujours majoritairement du secteur public : collectivités locales, établissements publics de coopération intercommunale, établissements publics fonciers et d'aménagements locaux, sociétés locales d'aménagement, mais aussi agences d'urbanisme et services de l'État (Dubus, 1999 ; Roux, 2003). La demande privée se limite à quelques entreprises propriétaires de foncier et aux grands groupes de promotion et de construction immobilière, qui interviennent en qualité d'opérateurs sur des projets d'urbanisme ou d'aménagement (Campagnac, 1992). Échaudés par la crise immobilière des années 1990, les rares aménageurs privés n’ont cependant guère le goût du risque pour lancer de grands programmes urbains (Roux, 2003). Les sociétés privées ne représentent ainsi que $5 \%$ des donneurs d'ordre du cabinet Codra, tandis que $70 \%$ de ses commandes de prestations émanent des collectivités locales, $15 \%$ des services de l'État et $10 \%$ d'organismes affiliés au secteur public ${ }^{14}$. La situation est analogue dans les autres cabinets de consultants : Interland n'affiche que trois commanditaires privés sur plus de 200 missions référencées depuis $2000^{15}$; le cabinet Tetra, une seule mission de programmation urbaine (pour la Régie Renault en 1996) sur plus de 300 références ${ }^{16}$.

Si la commande de conseils et d'études en stratégies urbaines est largement monopolisée par le secteur public, elle n'en est pas moins irrégulière et fortement dépendante du contexte général d'évolution des politiques urbaines.

Une première vague de commandes publiques correspond en effet au renouveau de la planification et des politiques urbaines, consécutive à la décentralisation des compétences d'urbanisme et d'aménagement aux communes et aux intercommunalités. À Lyon, elle se traduit notamment par l'élaboration d'un nouveau schéma directeur d'aménagement, qui mobilise l'ingénierie privée des cabinets Ten Conseil et Tetra, pour combler le manque de compétence de l'Agence d'urbanisme en gestion stratégique de projet, en communication et en prospective économique et territoriale. L'EIL apporte aussi son expertise pour amorcer la mise en place d'une politique économique métropolitaine. Ces collaborations permettent alors aux acteurs publics locaux de s'ouvrir aux nouvelles approches de l'urbanisme et de la planification stratégique et de se familiariser avec les enjeux du développement économique (Linossier, Menez, 2007).

La deuxième vague de commandes découle des lois Chevènement sur l'intercommunalité (1999), Voynet sur l'aménagement et le développement durable du territoire et Solidarité et Renouvellement Urbain (2000), qui ont relancé le marché du conseil et des études en stratégies et projets urbains. Les collectivités locales doivent refonder leur planification

\footnotetext{
${ }^{13}$ Expression empruntée à H. Huntzinger, assistant de recherche au Cerau (1968-1971), chargé d'études à la Sacec (1976-1977) et consultant fondateur de Tetra depuis 1980.

${ }^{14}$ http://www.codra-conseil.com

${ }^{15} \mathrm{http}: / /$ www.interland.info

${ }^{16}$ Tetra, Extrait de références, ronéo, 34 p.
} 
territoriale et leur réglementation du sol (Scot et PLU) ${ }^{17}$ et intégrer les enjeux de mixité sociale, de renouvellement et de durabilité dans leurs stratégies et projets urbains. Une demande nouvelle émerge en amont de l'action publique urbaine (études préalables, diagnostics, études de faisabilité), qui contribue à l'élargissement des commandes, tant sur des compétences opérationnelles (montage, programmation, commercialisation des projets) que sur des préoccupations de stratégie générale (Roux, 2003).

\subsection{Une demande fragmentée}

Les commandes publiques de conseil en stratégies et projets urbains sont cependant assez émiettées par rapport aux commandes formulées dans leurs autres domaines de compétences (services techniques par exemple), notamment au regard du montant moyen des marchés passés (Roux, 2003). Ce champ du conseil comprend en effet plusieurs « sous marchés », qui correspondent à différents besoins en matière de prestation de la part des commanditaires. Une impression de flou domine l'ensemble, tant les contours et les catégories sont difficiles à esquisser.

Le marché des études urbaines se situe en amont de l'action, lors de l'élaboration des documents réglementaires de planification ou de la préparation des programmes d'action. Il mobilise un large spectre de domaines d'expertise disciplinaires allant de la psychologie aux sciences de l'ingénieur. Celui de l'assistance à maîtrise d'ouvrage ou à maîtrise d'œuvre (AMO) accompagne la mise en œuvre de l'action, qu'il s'agisse de politiques thématiques comme les politiques de développement urbain, d'aménagement des espaces publics, de développement économique, de transport, de logement, culturelles, etc. ou d'opérations plus ponctuelles comme les projets urbains et la réalisation d'équipements collectifs. L'AMO s'est fortement développée au cours des dernières années, en raison du volontarisme croissant des communes et des intercommunalités en matière de développement territorial, de la multiplication et de la complexification des projets d'aménagement et de renouvellement urbain. Il fait appel à des compétences techniques ou méthodologiques particulières : programmation, montage et gestion de projets, techniques d'urbanisme et procédures d'aménagement, ingénierie, diagnostic, prospective, communication, concertation, évaluation, etc. Les marchés de la formation spécialisée, du management des organisations et du conseil informatique, juridique ou financier, se situent plutôt en marge des activités de conception ou de conduite des stratégies et politiques urbaines à proprement parler (Abiker, 1996). Ces différents «sous marchés » sont toutefois étroitement liés les uns aux autres et renvoient à des savoir-faire ou à des compétences spécifiques du côté des prestataires.

Parmi les différentes formes de marchés publics à la disposition des commanditaires publics, les formes fractionnées sont très fréquemment utilisées. Les marchés à lots (techniques, géographiques, etc.) permettent de répartir entre plusieurs prestataires potentiels les différentes tâches nécessaires à la réalisation d'une opération. Les marchés à bons de commande facilitent au contraire une certaine unité de traitement en confiant à un même prestataire plusieurs missions dans un même domaine d'intervention. Les marchés à tranches permettent quant à eux de conditionner et d'étaler dans le temps la réalisation des différentes phases d'un projet.

\footnotetext{
17 Schéma de cohérence territoriale et Plan Local d'Urbanisme. Ils remplacent les Schémas directeurs d'aménagement et d'urbanisme (Sdau) et les Plans d'occupation des sols (Pos).
} 


\subsection{Des montants de commandes peu élevés}

Le caractère émietté de la demande se répercute automatiquement sur les montants des commandes publiques de services en stratégies et projets urbains (études, conseils), qui sont relativement faibles. Le volume des dépenses d'études et de conseil d'urbanisme (hors maîtrise d'ouvrage) des collectivités locales est estimé entre 120 et 150 millions d'euros par an au début des années 2000 (Roux, 2003), ce qui représente environ 1,3\% de leurs budgets d'investissement.

Une grande partie des commandes formulées par les collectivités locales ou par leurs divers organismes « satellites » à dominante publique ${ }^{18}$ restent ainsi en dessous des seuils de publicité réglementée, établis par le Code des Marchés Publics (CMP) pour garantir l'égalité d'information des candidats ${ }^{19}$. Les commanditaires publics font cependant souvent du zèle quant à l'application du CMP, en rendant publics par les voies officielles prévues leurs appels et autres dialogues compétitifs, même si ceux-ci n'atteignent pas les montants seuils, afin de multiplier les candidats potentiels et ainsi augmenter leurs possibilités de choix entre plusieurs prestataires, malgré la relative rareté de l'offre. En outre, la faiblesse des sommes en jeu, notamment par rapport à d'autres domaines de passation de marchés publics (délégation de service public, attribution de grands chantiers d'équipement), semble préserver le champ du conseil en stratégies et projets urbains des dérives liées au financement des partis politiques ${ }^{20}$.

Le marché du conseil en urbanisme semble finalement souffrir d'un mal récurrent : le manque de crédits pour les études et le conseil (Audouin, 2008). L'existence et le développement d'une offre, qu'elle soit publique ou privée, sont très largement dépendants de l'évolution de la demande et des choix financiers publics. Quand les commandes se tarissent, cela remet en question l'existence même des BE et des cabinets de consultants. Toutefois, si la demande est actuellement soutenue par un contexte de renouvellement des politiques urbaines et territoriales en France, l'égalité d'accès à la commande de tous les prestataires présents sur le marché n'est pas pour autant garantie.

\section{Fragilité et monopolisation structurelles de l'offre de conseil}

La structure de l'offre présente des particularités qui contribuent à faire du marché du conseil en stratégies et politiques urbaines un marché atypique. L'offre s'organise en effet à partir de structures présentant des tailles et des statuts juridiques très variés, bien que relevant majoritairement du secteur privé : sociétés anonymes, coopératives, associations,

\footnotetext{
${ }^{18}$ Agences d'urbanisme, sociétés d'économie mixte, société publiques locales d'aménagement, établissements publics fonciers locaux, etc.

${ }^{19}$ En dessous de 90000 euros HT, la publicité doit être adaptée et réalisée par des moyens laissés au libre choix du commanditaire (presse, internet, courrier, etc.) ; entre $90000 \mathrm{HT}$ et les seuils communautaires (125 000,00 euros HT pour l'État ou 193000 euros HT pour les collectivités locales), la publicité est réglementée et doit se faire par annonce dans les bulletins officiels et journaux habilités au niveau local ; au-delà

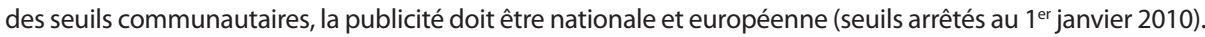
${ }^{20}$ Cette question n'a cependant pas été ouvertement abordée par l'auteur ni par les professionnels enquêtés lors de la recherche (Cf. Annexe méthodologique).
} 
professions libérales, organismes affiliés au secteur public, mais aussi laboratoires de recherche et établissements de formation supérieure relevant du secteur public. Le nombre de consultants privés dans le champ de l'urbain est estimé entre 1100 et 1600, auxquels s'ajoutent $30 \%$ de personnels « techniques » associés (secrétaires, infographistes, cartographes, vidéastes, plasticiens, etc.), soit un total d'environ 2000 personnes au maximum (Roux, 2003). Il s'agit d'un secteur d'activités extrêmement restreint voire confidentiel, mais au sein duquel la concurrence est rude et inégale en raison de la place occupée par le secteur public. Les agences d'urbanisme et autres services déconcentrés de l'Équipement bénéficient en effet d'une situation de quasi-monopole du côté de l'offre sur certaines activités et/ou territoires ${ }^{21}$. Les seconds tendent cependant à réduire progressivement leur intervention auprès des collectivités locales, à mesure que s'achève le processus de décentralisation.

\subsection{Quelles lignes de partage de l'offre?}

L'offre est majoritairement constituée d'une pluralité de petites structures individuelles ou de deux à cinq personnes organisées autour d'un consultant confirmé, souvent architecte. Elles ont toutefois beaucoup de difficulté à exister et à se maintenir face à la concurrence exercée par les gros cabinets et par certains organismes affiliés au secteur public, car ces derniers bénéficient de conditions d'accès et de réponse aux commandes d'études et de conseils en stratégies et projets urbains très avantageuses, qui génèrent une certaine distorsion des conditions de mise en concurrence des prestataires sur le marché.

Les gros cabinets de plus de vingt salariés ou collaborateurs associés sont rares, tout au plus une dizaine en France (Codra et Interland atteignent tout juste ce seuil). Ils correspondent notamment à des composantes de grands cabinets de conseil et d'audit internationaux généralistes (KPMG, Ernst \& Young, etc.), qui ont développé une branche d'activités «Secteur public » pour répondre à la demande des collectivités locales et de l'État en matière d'organisation, de gestion des compétences, d'efficacité des politiques publiques, de gouvernance, de management de projets complexes, etc. Ces grands cabinets généralistes qui investissent le champ de l'urbain et des territoires le font généralement en «noyant » leurs petites équipes dédiées dans des départements « secteur public » ou d'AMO dans le bâtiment et les travaux publics (Roux, 2003). Le Lyonnais Algoé Consultants fait figure d'exception dans ce paysage, en affichant un savoir-faire spécifique en gestion des grands projets complexes d'équipement pour le compte de commanditaires publics ${ }^{22}$, à travers sa filiale Aldev puis son département «Stratégie et territoire ». Depuis 2001, il a réorganisé ses services par unités de clientèles sur le même modèle que ses concurrents internationaux, mais continue à afficher ses prestations par métiers ${ }^{23}$.

Une tendance se dégage, distinguant ce qui relève de l'urbanisme réglementaire et opérationnel (les actions de détail), plutôt pris en charge par les petits cabinets d'architecture

\footnotetext{
${ }^{21}$ Notamment l'élaboration des documents de planification urbaine et réglementaire : Scot et PLU dans les zones rurales, Plans des Déplacements Urbains et Programmes Locaux de l'Habitat dans les petites agglomérations.

${ }^{22}$ Depuis les années 1970, Algoé a notamment organisé l'aménagement de plusieurs stations de sports d'hiver, du Marché d'intérêt national de Rungis, de l'Université technologique de Compiègne, de la Cité des Sciences de la Villette, d'infrastructures de transports, des Jeux Olympiques d'Albertville, etc.

${ }^{23} \mathrm{http} / / / w w w . a l g o e . f r$
} 
et d'urbanisme, de ce qui relève des stratégies et projets urbains (les politiques urbaines et les programmes d'opérations complexes), plutôt pris en charge par de grands cabinets de conseil en gestion et en stratégie. Le cabinet Algoé Consultants, avec ses dizaines de collaborateurs sur les questions urbaines et territoriales, contredit cependant encore cette affirmation, en intervenant aussi bien sur des missions stratégiques et de gestion de projets urbains complexes (élaboration et pilotage de schémas de développement d'agglomération par exemple) que sur des missions plus basiques d'AMO opérationnelle (réalisation de calendriers de phasage technique pour les opérations d'urbanisme). À l'inverse, des consultants libéraux interviennent en qualité de conseillers techniques et stratégiques sur des missions de grande ampleur, concernant la définition, l'organisation et le suivi de politiques urbaines ou la conception et l'assistance à maîtrise d'ouvrage de projets urbains complexes (J.-Y. Chapuis, J.-P. Charbonneau, J.-M. Roux). Ils le font le plus souvent en s'associant avec un « grand nom » de l'urbanisme et avec des partenaires complétant leurs compétences techniques (sociétés d'ingénierie spécialisée notamment).

La distinction entre les études amont liées à la prospective, à la stratégie et à la planification et les prestations liées à des projets urbains opérationnels met ainsi en évidence quelques grandes lignes de structuration de l'offre, autour de grands cabinets de consultants, qui s'attachent, en fonction de la demande, des compétences en urbanisme, en architecture, en développement économique ou en ingénierie technique spécialisée, et une multitude de petits cabinets ou de professionnels exerçant en libéral. Les associations entre équipes appartenant à des cabinets différents et les montages parfois complexes faisant intervenir plusieurs structures (par le biais d'un ou deux collaborateurs de chaque) sont très fréquents, bien qu'ils soient la plupart du temps circonstanciels et à géométrie variable selon les commandes. La co-traitance et la sous-traitance sont particulièrement développées dans le cadre des réponses à appel d'offres sur des projets d'aménagement ou d'urbanisme complexes. La capacité à nouer des partenariats plus ou moins réguliers et pérennes en fonction du type de demande constitue d'ailleurs un argument commercial mis en avant par les cabinets de conseil en stratégies et projets urbains dans leurs supports de communication, à travers l'établissement de listes de partenaires classés par compétence ou par type de prestation.

Des comportements analogues sont observés dans les secteurs d'activités voisins de l'évaluation des politiques urbaines, de la recherche-action et de la participation, avec la constitution de « collectifs » de consultants - chercheurs relativement spécialisés dans les domaines du développement territorial et de la Politique de la Ville, autour de la coopérative Acadie et du groupe Reflex notamment ${ }^{24}$.

Les consultants indépendants et les cabinets privés jouent également quasiment tous la carte de la pluridisciplinarité. Cette stratégie de diversification des compétences, en interne ou en association avec d'autres consultants, des cabinets d'architecture et des bureaux d'études techniques spécialisés, renforce la capacité des petites structures ou des professions libérales à répondre aux besoins des commanditaires publics aux différents stades de la conception des projets, de la réflexion stratégique en amont à l'AMO en aval, en offrant une prestation

\footnotetext{
${ }^{24}$ http://www.acadie-reflex.org

Voir Tissot S., 2002, Réformer les quartiers. Enquête sociologique sur une catégorie de l'action publique, Thèse pour le Doctorat en Sociologie, EHESS et Nonjon M., 2006, Quand la démocratie se professionnalise. Enquête sur les experts de la participation, Thèse pour le Doctorat en Science Politique, Université Lille 2.
} 
complète de conduite des stratégies et des projets urbains. La polyvalence est un argument de fiabilité et de compétence, de maîtrise du champ d'activités dans sa globalité. Elle est donc aussi mise en avant dans les stratégies d'affichage et de promotion commerciale.

\subsection{La « chasse gardée » des agences d'urbanisme}

Les agences d'urbanisme prolongent le système d'expertise mis en place par l'État pour accompagner la politique d'aménagement du territoire et d'urbanisation. Elles participent à l'émergence d'un pouvoir d'expertise local en matière d'urbanisme, grâce aux transferts de personnels et de savoir-faire opérés depuis les services de l'Équipement ${ }^{25}$ et les BE précédemment évoqués, et au recrutement de nombreux spécialistes (sociologues, économistes, juristes, ingénieurs, graphistes, etc.).

Ces organismes affiliés au secteur public ont généralement un statut privé d'association loi 1901 (Danan, 1976 ; Prévot et alii, 2008). Leurs membres sont des entités publiques ayant des compétences ou des implications en aménagement et en urbanisme (État et collectivités locales principalement). Elles sont représentées par la Fédération nationale des Agences d'Urbanisme $\left(\right.$ FNAU) ${ }^{26}$. En tant que maîtres d'œuvre d'études urbaines, elles réalisent la majeure partie des travaux d'expertise nécessaires en amont de la conduite des politiques urbaines, pour les établissements publics de coopération intercommunale ${ }^{27}$, les municipalités et les divers organismes publics qui les financent, dans le cadre de programmes partenariaux propres à chaque configuration locale d'agglomération. Leurs équipes et leurs savoirs sont constitués en fonction des besoins d'études de leurs commanditaires, qui sont principalement leurs membres financeurs, pour un effectif salarié total d'environ 1200 personnes (Roux, 2003).

Les agences d'urbanisme occupent donc de fait une place privilégiée dans le champ des études et du conseil en stratégies et projets urbains, grâce aux missions qui leur sont confiées « en direct » par les membres adhérents dans le cadre du programme partenarial, c'est-à-dire en dehors de toute logique de marché et de mise en concurrence avec d'autres prestataires potentiels. Elles bénéficient ainsi d'une véritable « chasse gardée » en matière d'études, notamment concernant l'élaboration des documents de planification intercommunaux. Leur statut associatif public est censé garantir leur impartialité et leur capacité à développer des approches globales des problématiques urbaines.

Dans l'agglomération lyonnaise par exemple, le volant d'études du Grand Lyon ${ }^{28}$ est réparti à peu près équitablement entre l'Agence d'urbanisme et les cabinets privés. Cette répartition s'articule autour de leurs champs de compétences respectifs : l'Agence s'occupe des volets stratégiques des politiques urbaines et de la planification territoriale, qui nécessitent une vision d'ensemble des enjeux territoriaux (schéma de développement économique - SDE, schéma de développement universitaire, schéma d'accueil des entreprises, Scot, etc.), tandis que les cabinets privés interviennent principalement sur les études pré-opérationnelles, les études préalables et la conduite des projets urbains. Sur certaines missions très spécifiques,

\footnotetext{
25 Plusieurs ingénieurs des Ponts et Chaussées se voient confier la direction des agences d'urbanisme, qui sont en grande partie financées par l'État.

${ }^{26}$ Association regroupant 52 organismes publics d'étude et de réflexion sur l'aménagement et le développement des grandes agglomérations urbaines françaises (http://www.fnau.org/decouvrir/presentation-fnau.asp)

${ }^{27}$ Communautés de communes, communautés d'agglomération et communautés urbaines.

${ }^{28}$ Communauté urbaine de Lyon.
} 
qui nécessitent des compétences situées en marge de son cœur de métier, l'Agence s'appuie sur l'AMO et l'expertise de cabinets extérieurs (Algoé Consultants pour l'élaboration du SDE ; Ten Conseil et Tetra, entre autres, pour l'élaboration du Schéma directeur Lyon 2010 ; L'EIL pour la mise en place d'un observatoire économique, etc.).

Les agences n'ont cependant recours à la sous-traitance que de façon occasionnelle et ponctuelle, uniquement lorsqu'elles ne possèdent pas en interne les compétences techniques ou l'expertise nécessaire pour réaliser elles-mêmes certaines missions. Elles n'ont pas la capacité financière suffisante pour sous-traiter de façon massive leurs missions d'études à des prestataires spécialisés, ni pour se positionner comme des structures de coordination des tâches entre les collectivités publiques donneuses d'ordre et les prestataires privés.

\subsection{Une offre publique qui fausse la concurrence}

Presque la moitié des agences investissent aussi le marché concurrentiel de l'offre d'études et du conseil, indépendamment de leur programme partenarial, répondant aux appels des collectivités locales ou des organismes publics ne faisant pas partie de leurs adhérents. Elles sont alors en concurrence directe avec les cabinets de consultants et les bureaux d'études privés, mais contrairement à eux, elles sont en capacité de facturer leurs prestations à prix coûtant - leurs frais fixes étant financés par leurs partenaires - ce qui leur permet de proposer des tarifs très compétitifs.

Un accord-cadre conclu entre l'État et la FNAU plafonne cependant la part des prestations réalisées en dehors du programme partenarial (ou contrats spécifiques) à $20 \%$ des recettes de chaque agence, pour réaffirmer leur caractère non lucratif et le respect d'une certaine déontologie publique interdisant de «tuer» l'offre privée. Les agences de Lyon et de Bordeaux, parmi les plus actives, limitent pour l'instant leur volet de contrats spécifiques à $5 \%$ du montant de leurs recettes, mais leurs directeurs participent aux discussions conduites au sein de la FNAU concernant le risque de désengagement financier de certains partenaires (l'État notamment) et les possibilités de développer leurs prestations sur le marché concurrentiel, pour pallier le rétrécissement des budgets. L'agence d'urbanisme bordelaise A'Urba brouille d'ailleurs les repères entre secteur public et marché privé, en se présentant comme une «véritable entreprise publique de matière grise ${ }^{29}$.

Des laboratoires de recherche et des instituts universitaires de formation supérieure (Instituts d'urbanisme de Paris, de Lyon, de Grenoble, etc.) interviennent également en qualité de prestataires sur le marché des études et du conseil en urbanisme, à des prix très bas et parfois à titre gracieux. Ils réalisent des travaux d'études et de programmation pour le compte des collectivités locales et des agences d'urbanisme, dans le cadre de contrats de recherche-action, d'ateliers et de stages professionnels de master ou de conventions Cifre pour la préparation de thèses de doctorat ${ }^{30}$. De statut public, ils sont généralement rattachés à une université ou à une grande école. Certains comme L'EIL (devenu Lab'Urba) ont créé des associations à but non lucratif pour faciliter la gestion des contrats

\footnotetext{
${ }^{29} \mathrm{http}: / /$ www.aurba.com/site/agence_generalites.html

${ }^{30}$ Voir notamment Prévot M. et Simard P., « L'expertise des agences d'urbanisme au miroir de la recherche. Quelques initiatives de recherche et de réflexion récentes ", Les Annales de la Recherche Urbaine, $\mathrm{n}^{\circ} 104$, juin 2008, pp. 84-94.
} 
marchands conclus à titre onéreux, mais cette alternative, à la limite de la légalité, a été remise question par la Cour des Comptes.

Les agences d'urbanisme, les instituts de formation et les laboratoires publics de recherche occupent ainsi une position avantageuse dans la captation des contrats auprès des collectivités locales et de l'État, car ils disposent de moyens permanents (budget, main-d'œuvre) leur permettant d'assurer leurs missions principales, indépendamment de la facturation des prestations de conseil ou d'études, et pour les premières d'un accès direct à une grande partie de la demande à travers les programmes partenariaux.

Leur présence comme prestataires de services du côté de l'offre biaise le fonctionnement concurrentiel du marché des études et du conseil en stratégies et projets urbains, en créant des différences de tarification et d'accès aux commandes, très pénalisantes pour les petits cabinets de conseil privés. Cette situation est toutefois appelée à évoluer en raison de la transposition en France des directives européennes relatives à l'obligation de mise en concurrence des marchés publics depuis 2005, du moins pour les montants de commande les plus importants.

\section{Les ressources des structures privées pour capter les marchés}

L'Association des Consultants en Aménagement et Développement des territoires $(\text { Acad })^{31}$ tente de pallier la forte atomicité de l'offre et la pression concurrentielle du champ d'activités, en fédérant un réseau national de compétences professionnelles pour assurer la promotion commerciale des petites entreprises privées du secteur. Elle génère une dynamique d'interconnaissance, sur laquelle s'appuient les professionnels pour établir leurs relations de co-traitance et de sous-traitance, mais les partenariats se développent également en dehors de cette organisation collective.

Les grands cabinets sont cependant absents de cette organisation, à l'exception de Codra ${ }^{32}$. De la même façon, certaines figures du conseil en stratégies et projets urbains, exerçant en libéral ou au sein de cabinets, n'adhèrent pas à l'Acad (J.-P. Charbonneau, J.-Y Chapuis, J. De Courson, F. Ampe, Tetra, etc.). Il semble donc qu'en matière d'offre de conseil, certains aient besoin d'une vitrine et d'un support de visibilité dans la recherche de clients, alors que d'autres bénéficient de ressources leur permettant d'accéder à la commande par ailleurs. Quelles sont ces ressources spécifiques dont bénéficient certains consultants ?

\subsection{Les logiques de niches}

Quelques cabinets proposent des démarches innovantes, qui consistent à accompagner les collectivités dans la réalisation de leurs projets lorsque ceux-ci s'avèrent complexes à définir et à piloter. Les consultants assurent alors un rôle de coordination, d'intégration et de médiation entre tous les acteurs mobilisés (élus, fonctionnaires et techniciens, collectivités

\footnotetext{
${ }^{31}$ Elle rassemble une centaine de cabinets indépendants de conseil, dont la grande majorité compte moins de dix salariés ou collaborateurs associés, spécialisés dans les domaines de l'urbanisme réglementaire, de I'AMO en aménagement et urbanisme, du développement durable et du développement économique local. ${ }^{32}$ Certains adhèrent à Syntec Ingénierie ou à Syntec Management, qui couvrent des champs plus vastes du conseil.
} 
associées, établissements publics, services déconcentrés de l'État, investisseurs privés, etc.), tels des «accoucheurs de projets » (Abiker, 1996, p. 26). Ils se créent ainsi des rentes de situation, en occupant des positions de niche sur des marchés locaux, ou sur des secteurs d'activités spécifiques au sein du champ, à l'échelle plurirégionale voire nationale.

Depuis les années 1990, le cabinet Algoé Consultants domine par exemple le marché de l'AMO en stratégies et projets urbains dans l'agglomération lyonnaise. Il est mandataire de plusieurs marchés publics du Grand Lyon, dont certains à bons de commande, concernant le développement économique et la conduite d'opérations d'urbanisme complexes, et laisse peu de place aux éventuels concurrents. Il est notamment passé maître dans l'animation et le pilotage des projets d'urbanisme, en proposant des tableaux de bord et des plannings opérationnels très élaborés. Les marchés de développement économique que le cabinet a obtenus dans d'autres territoires français (Grenoble, Pays Roannais, Marseille, etc.) s'inscrivent également dans la continuité de la mission d'AMO du SDE de l'agglomération lyonnaise réalisée pour l'Agence d'urbanisme de Lyon, qui repose sur une méthodologie stratégique particulièrement performante (enquête, diagnostic, plateforme stratégique).

Cette situation avantageuse est un héritage du travail pionnier du Groupe Ten Conseil, fondé par C. Neuschwander puis racheté par Algoé Consultants. Ten a pris son essor au moment où les grands BE liés à la sphère étatique périclitaient, en s'orientant vers le traitement des problèmes économiques et urbains qui se posaient désormais aux collectivités locales. Sa filiale rhônalpine a notamment élaboré une nouvelle conception stratégique de l'urbanisme, privilégiant le développement du contenu économique et urbain des projets et la collaboration avec les investisseurs privés, applicable du quartier à la région urbaine, plutôt qu'une simple approche par la forme. Le savoir-faire novateur de Ten en termes de projet urbain a été intégré aux compétences historiques d'Algoé Consultants en matière de gestion des projets complexes, lui assurant une véritable rente de situation à l'échelle locale en matière d'urbanisme opérationnel et une niche de marché régionale voire nationale pour le développement économique stratégique.

Cet exemple reflète l'importance de certaines ressources, comme l'antériorité de la présence sur le marché et la reconnaissance de l'expérience acquise qui en découle, pour permettre à certains consultants d'accéder aux marchés et d'obtenir des commandes plus facilement que d'autres.

\subsection{L'appartenance à des réseaux socioprofessionnels ou politiques}

Des relations durables entre consultants ou avec des représentants de la sphère publique de la commande se nouent sur des scènes plus informelles que l'Acad, par exemple celles de la socialisation étudiante et du militantisme politique. Les consultants ont des cursus de formation assez homogènes. Plusieurs sont issus de grandes écoles d'ingénieurs (Ecole Centrale des Arts et Manufactures de Paris notamment) et la plupart affichent des profils de formation initiale pluridisciplinaires en sciences humaines et sociales, avec une ouverture à l'économie prononcée (Cf. Tableau 2, Annexe méthodologique).

Des logiques d'interconnaissance et de réseaux en découlent, qui se retrouvent dans les dynamiques de collaboration, de co-traitance ou d'accès à la commande. Le corps des ingénieurs centraliens constitue notamment un vivier de personnels pour les cabinets de consultants en stratégies et projets urbains. C. Neuschwander s'est ainsi appuyé sur ses 
réseaux de socialisation issus de l'École Centrale de Paris, de la Jec et de l'Unef ${ }^{33}$, pour développer et animer les différents cabinets de conseil qu'il a créés (Groupe Ten, EDR, Agence de Notation des Villes, Métropoles et Territoires). Plus largement, la relative unité des profils de formation et un « capital » militant commun renforcent le sentiment de partager une même culture et facilitent les rapprochements professionnels entre consultants de même génération ou de générations différentes, ainsi qu'avec leurs homologues de formation travaillant au sein des collectivités publiques commanditaires ${ }^{34}$.

La proximité avec le monde politique et la sphère de la demande publique est également une ressource importante des consultants pour accéder aux commandes. La plupart des professionnels du conseil cultivent leurs relations avec les élus et les cadres des collectivités locales, quand ils ne s'inscrivent pas eux-mêmes dans ces groupes d'acteurs. Une grande partie des contrats publics de Ten et EDR Conseils ont ainsi été signés grâce à la mobilisation des réseaux politiques de $\mathrm{C}$. Neuschwander, notamment au sein de la mouvance socialiste dont il est proche (Neuschwander, 2011). Certains consultants sont ou ont été des élus locaux à responsabilité (F. Ampe a été maire de Chambéry, J.-Y. Chapuis est élu à Rennes depuis 1983), d'autres ont dirigé des cabinets politiques.

Des relations étroites avec la sphère de la décision confèrent aux consultants une connaissance fine des enjeux politiques de l'action publique et la capacité de se positionner en empathie avec les maîtrises d'ouvrage. Cependant, elles font aussi planer les soupçons de corruption et de favoritisme dans l'attribution des marchés (De Courson, 2008).

\subsection{Pluriactivité et valorisation de l'expérience}

Le cumul de plusieurs activités est une pratique courante dans le monde du conseil (Henry, 1992; De Montlibert, 2007). Dans le temps ou simultanée, elle constitue un atout commercial pour les consultants et leur permet de nouer des relations étroites avec leurs concurrents et partenaires potentiels, ainsi qu'avec leurs donneurs d'ordre. La pluriactivité est facilitée dans le champ des stratégies et projets urbains par une grande perméabilité du marché du travail, entre secteur public et secteur privé, entre sphère de l'offre et sphère de la demande et entre domaine des études stratégiques et domaine de l'opérationnel, qui est héritée du système technocratique d'expertise de l'État.

Plusieurs consultants exercent en parallèle des fonctions d'enseignant-chercheur ou de professeur associé (Cf. Tableau 2, Annexe méthodologique). Ils tirent plusieurs bénéfices de cette double activité. Elle leur assure d'abord un revenu complémentaire aux revenus tirés du conseil, voire un revenu de substitution dans les périodes de repli de la demande. Elle consolide ensuite leur crédibilité vis-à-vis des commanditaires publics et leur ouvre des perspectives de commandes grâce aux contacts noués au sein des écoles d'ingénieurs et des instituts d'urbanisme avec de futurs cadres administratifs ou territoriaux. Au-delà, elle renforce les dynamiques d'échanges de modèles et de circulation des idées entre les praticiens confirmés et les jeunes professionnels, entre le monde de l'enseignement et de la recherche et le monde de la pratique, contribuant ainsi à la construction d'une culture professionnelle et à l'établissement de valeurs communes spécifiques au champ de l'urba-

\footnotetext{
${ }^{33}$ Jeunesse Étudiante Chrétienne et Union Nationale des Étudiants de France.

${ }^{34}$ Plusieurs d'entre eux sont membres de l'ONG Urbanistes du Monde, créée en 2005 par J. De Courson.
} 
nisme et de l'aménagement. Un constat analogue peut être fait concernant leur propension à publier des ouvrages et des articles dans des revues spécialisées ${ }^{35}$.

Le contexte fortement concurrentiel et incertain du marché (irrégularité de la demande, précarité des situations professionnelles), mais aussi des stratégies plus personnelles (recherche de nouvelles responsabilités ou de meilleures rémunérations), encouragent les consultants à envisager un ou plusieurs changements de statut et d'employeur au cours de leur carrière. Les trajectoires de J. De Courson et de J.-M. Roux reflètent bien cette propension à la mobilité professionnelle. Le premier a commencé sa carrière à l'Agence d'urbanisme de Lille puis au Service Technique de l'Urbanisme du ministère de l'Équipement comme contractuel. Il a été directeur adjoint de l'Agence d'urbanisme de Lyon au moment de l'élaboration du SD Lyon 2010, avant de finir sa carrière comme consultant chez Ten et Algoé. Le second a démissionné de la fonction publique en 1970 pour monter le cabinet Area. Il a ensuite intégré le cabinet Codra et fait un bref passage dans la filiale d'aménagement urbain d'un grand groupe privé, avant de mettre ses compétences opérationnelles au service de la Société Centrale d'Équipement du Territoire pendant 12 ans. Il est finalement retourné au conseil en créant le cabinet Transversal.

Ces parcours professionnels, faits du cumul d'activités d'enseignement et de pratique, d'une succession de passages d'une position à une autre, d'une structure à une autre et d'allers-retours entre secteur privé et secteur public, reflètent l'importance de l'expérience accumulée et de la bonne connaissance des différents versants du marché pour pouvoir s'établir comme consultant en stratégies et projets urbains : offre / demande, public / privé, études stratégiques / pilotage opérationnel, formation / pratique, etc.

Au final, ils montrent aussi que la position de consultant en stratégies et projets urbains, notamment en profession libérale, est plutôt une position de fin de carrière, qui repose sur la valorisation de l'expérience acquise dans le champ de l'urbanisme et de l'aménagement. L'antériorité de la présence sur le marché - que ce soit du côté de la commande en qualité d'élu ou de salarié de la puissance publique comme du côté de l'offre en tant que chargé d'études au sein d'un cabinet de conseil - représente un avantage indéniable pour les consultants, car elle augmente leurs chances de nouer des relations d'interconnaissance du côté de l'offre et de la demande, de pouvoir afficher des références et de bénéficier d'une certaine réputation, afin d'accéder plus facilement aux commandes.

\section{Conclusion}

Les travaux sur le conseil en gestion auprès des administrations publiques pointent le rôle assez important joué par les grands cabinets dans la restructuration des services publics et l'évolution de la conduite des politiques publiques en France, particulièrement au niveau local. Cette influence refléterait le passage d'un système d'action publique technocratique à ce que certains nomment la « consultocratie» (Saint Martin, 2001). L'analyse du champ du conseil en stratégies et projets urbains relativise quelque peu cette affirmation, en révélant plutôt l'existence d'un marché atypique, au sein duquel l'espace occupé par le secteur public est encore très étendu et les héritages du système technocratique sont encore prégnants. Cette

${ }^{35}$ Urbanisme, Le Moniteur, Diagonal, Études foncières, Les Annales de la Recherche Urbaine, etc. 
domination du secteur public sur la demande et sur l'offre induit une certaine précarité des positions et un accès à la commande difficile pour les cabinets de conseil.

En raison des caractéristiques intrinsèques de ce marché atypique, les carrières des consultants s'effectuent le plus souvent à cheval sur plusieurs univers professionnels liés à l'urbanisme et à l'aménagement. Ces logiques d'échange entre le secteur public et le secteur privé, entre sphère politique et sphère technique ou entre monde académique et monde de la pratique sont largement hérités de l'époque où de grands bureaux d'études alimentaient le pouvoir d'expertise technocratique de l'État. Ils se poursuivent aujourd'hui autour de la satisfaction des besoins d'expertise des collectivités locales et assurent aux consultants les ressources nécessaires pour accéder aux commandes publiques. Ils invitent à dépasser l'impression première de déséquilibre structurel, dégagée par l'observation du fonctionnement de ce marché, pour envisager au contraire l'intensité et le caractère indispensable des relations marchandes et non-marchandes existant entre la commande publique et les consultants privés, car elles rendent possibles la circulation des idées, l'établissement d'une culture commune et la formulation de réponses adaptées aux enjeux contemporains des villes. Les savoir-faire, les méthodes, les approches innovantes, la meilleure lisibilité, la crédibilité, la souplesse d'intervention ou plus simplement les compétences qu'ils apportent à leurs clients - responsables politiques et services techniques des organismes publics locaux - constituent ainsi des arguments probants en faveur de leur maintien et de leur développement, au service de l'action publique. 


\section{Bibliographie}

ABIKER D., (1996). Les consultants dans les collectivités locales, LGDJ, Paris.

AUDOUIN J., (2008). Rémunération des études urbaines : un dangereux discrédit, D'Architectures 176, 51-75.

BARDET F., (2005). Institution des expertises urbaines dans la construction de l'action publique. Retour sur la métamorphose urbaine lyonnaise dans les années 1960 et 1970, Rapport pour le PUCA / Ministère de l'Équipement.

BECKER H., (1985). Outsiders. Études de sociologie de la déviance, Métailié, Paris.

BERREBI-HOFFMANN I., GRÉMION P. (2009), Élites intellectuelles et réforme de l'État. Esquisse en trois temps d'un déplacement de l'expertise, Cahiers internationaux de Sociologie 126, 39-59.

CAMPAGNAC E., (1992). Les grands groupes de la construction : nouveaux acteurs urbains, L'Harmattan, Paris.

CLAUDE V., (2006). Faire la ville. Les métiers de l'urbanisme au XX'e siècle, Parenthèses, Marseille.

DANAN Y.-M., (1976). Les agences d'urbanisme d'agglomération, Centre de Recherche d'Urbanisme, Paris.

DE COURSON J., (2008). Le goût du pouvoir, L'Harmattan, Paris.

DE MONTLIBERT C., (2007). Les agents de l'économie. Patrons, banquiers, journalistes, consultants, élus. Rivaux et complices, Raisons d'agir, Paris.

DUBUS T., (1999). L'évolution du marché des études dans le domaine de l'aménagement, Notes du CPVS, Ministère de l'Équipement, Paris.

HENRY O.,(1992). Entre savoir et pouvoir : les professionnels de l'expertise et du conseil, Actes de la Recherche en Sciences sociales $95,37-54$.

LINOSSIER R., MENEZ F., (2007). L'Agence d'urbanisme de Lyon : avant-garde et creuset d'expertise pour une politique urbaine stratégique, Territoire en mouvement 2, 57-71.

NEUSCHWANDER C., (2011). Claude Neuschwander. Une vie de militance(s), Adels / Ed. Yves Michel, Paris.

PRÉVOT M., (2007). Les agences d'urbanisme en France : l'expérimentation, l'innovation et leurs limites, Territoire en mouvement, $\mathrm{n}^{\circ} 2$.

PRÉVOT M. et al., (2008). Les agences d'urbanisme en France. Perspectives de recherche pluridisciplinaires et premiers résultats autour d'un nouvel objet, Métropoles, $\mathrm{n}^{\circ} 3$, en ligne http://metropoles.revues.org/2322

REGGAZZOLA T.,(1988). Recherche autour d'une amnésie. L'action des bureaux d'études au cours de la période 1960 et 1970, Association Orélie, Rapport pour la DRI / Ministère de l’Urbanisme et du Logement, Paris.

ROUX J.-M., (2003). Les études urbaines : budgets disponibles, prix de revient des consultants, conditions de leur mise en concurrence, Rapport pour la DGUHC / Ministère de l'Équipement, Scet Projets urbains, Paris.

SAINT MARTIN D., (2001). Les cabinets de conseil et la "remarchandisation" de la politique sociale dans les État-providence de type libéral, Lien social et Politique 45, 131-144. 


\section{Annexe méthodologique}

Cet article résulte de l'étude des trajectoires professionnelles (ou « carrières ») d'un échantillon de professionnels de l'urbanisme, de l'aménagement et du développement local, qui exercent ou ont exercé une activité de consultant ou de chargé d'études dans le champ du conseil en stratégies et projets urbains en France, menée entre fin 2009 et début 2011. Elle s'inscrit dans l'axe de travail $n^{\circ} 1$ - portant sur les personnels des agences d'urbanisme, les bureaux d'études et les membres des Conseils de développement - du programme de recherche CHRISTIANA «De l'urbanisme au développement : des militants d'origine chrétienne dans la fabrique de la ville de 1960 à nos jours (France / Belgique) », dirigé par Maryvonne Prévot pour le compte de la MESHS USR 3185/CPER Nord-Pas-de-Calais 2009-2010.

L'analyse croise :

- une approche structurelle des cabinets de conseil, à partir d'une douzaine d'études de cas (Tableau 1);

- une sociographie des consultants en stratégies et projets urbains, à partir de l'étude de onze parcours individuels de carrière : cursus de formation, engagements professionnels et militants, étapes de carrière, positionnement par rapport aux structures, etc. La plupart ont été investigués par entretiens et recherches documentaires, sauf les noms suivis de (D), qui ont été uniquement investigués par voie documentaire (Tableaux 1 et 2).

Les recherches bibliographiques (lecture de rapports, publications) ont permis de cerner l'activité des bureaux d'études et des cabinets de conseils en urbanisme, aménagement et développement local en France depuis les années 1950, les investigations documentaires sur Internet (exploration des sites des cabinets de conseil, collecte d'éléments biographiques et des curriculum vitae des consultants) et la réalisation de quinze entretiens avec des professionnels du secteur (Tableau 3) ont permis de dégager les modalités de fonctionnement du marché du conseil en stratégies et projets urbains. 
Tableau 1 : Présentation de l'échantillon

\begin{tabular}{|c|c|c|c|c|c|}
\hline Nom du cabinet & Acronyme & Statut & $\begin{array}{l}\text { Date de } \\
\text { création }\end{array}$ & $\begin{array}{l}\text { Date de } \\
\text { disparition }\end{array}$ & $\begin{array}{l}\text { Consultants } \\
\text { investigués }\end{array}$ \\
\hline $\begin{array}{l}\text { Bureau d'études et } \\
\text { de réalisations urbaines }\end{array}$ & BERU & $\begin{array}{l}\text { Société } \\
\text { coopérative }\end{array}$ & 1957 & $\begin{array}{l}1977 \\
\text { (transformé } \\
\text { en Codra) }\end{array}$ & B. Archer \\
\hline $\begin{array}{l}\text { Société d'assistance } \\
\text { au commerce. Études } \\
\text { et conseils }\end{array}$ & SACEC & SARL & 1950 's & 1978 & H. Huntzinger \\
\hline $\begin{array}{l}\text { Association lyonnaise de } \\
\text { gestion et d'organisation } \\
\text { des entreprises }\end{array}$ & ALGOE & $\begin{array}{l}\text { SARL puis } \\
\text { Société } \\
\text { coopérative }\end{array}$ & 1959 & & $\begin{array}{l}\text { J. De Courson } \\
\text { D. Gaudron } \\
\text { C. Cizeron }\end{array}$ \\
\hline $\begin{array}{l}\text { Centre de recherches } \\
\text { et d'études sur } \\
\text { l'aménagement urbain }\end{array}$ & CERAU & Filiale CDC & 1967 & $\begin{array}{l}1971 \\
\text { (absorbé } \\
\text { par Beture) }\end{array}$ & $\begin{array}{l}\text { H. Huntzinger } \\
\text { R. Prud'homme }\end{array}$ \\
\hline $\begin{array}{l}\text { Atelier de recherches } \\
\text { et d'études sur } \\
\text { l'aménagement }\end{array}$ & AREA & $\begin{array}{l}\text { SARL puis } \\
\text { société } \\
\text { coopérative }\end{array}$ & 1970 & 1977 & J.-M. Roux \\
\hline $\begin{array}{l}\text { Ten Conseil } \\
\text { Développement local, } \\
\text { communication }\end{array}$ & TEN & $\begin{array}{l}\text { Société } \\
\text { coopérative }\end{array}$ & 1976 & $\begin{array}{l}1995 \\
\text { (absorbé } \\
\text { par Algoé) }\end{array}$ & $\begin{array}{l}\text { C. Neuschwander } \\
\text { J. De Courson } \\
\text { D. Gaudron }\end{array}$ \\
\hline $\begin{array}{l}\text { Conseil à la décision } \\
\text { et à la réalisation en } \\
\text { aménagement }\end{array}$ & CODRA & SARL & $\begin{array}{l}1977 \\
\text { (remplace } \\
\text { Béru) }\end{array}$ & & J.-M. Roux \\
\hline $\begin{array}{l}\text { Techniques, études } \\
\text { et recherches en } \\
\text { aménagement }\end{array}$ & TETRA & SARL & 1980 & & H. Huntzinger \\
\hline \multirow[t]{2}{*}{$\begin{array}{l}\text { Laboratoire } \\
\text { d'observation de } \\
\text { l'économie et des } \\
\text { institutions locales }\end{array}$} & L'CEIL & $\begin{array}{l}\text { Laboratoire } \\
\text { universitaire } \\
\text { de recherche/ } \\
\text { association }\end{array}$ & 1980 & $\begin{array}{l}2000 \\
\text { (fondu dans } \\
\text { CRETEIL } \\
\text { et Lab'Urba) }\end{array}$ & R. Prud'homme \\
\hline & & $\begin{array}{l}\text { Profession } \\
\text { libérale }\end{array}$ & 1989 & & $\begin{array}{l}\text { J.-P. Charbonneau } \\
\text { (D) }\end{array}$ \\
\hline $\begin{array}{l}\text { INterland - Agence } \\
\text { d'architecture, } \\
\text { urbanisme et paysage }\end{array}$ & INterland & SARL & 2000 & & (D) \\
\hline $\begin{array}{l}\text { Entreprises et } \\
\text { Développement } \\
\text { Régional-Conseils }\end{array}$ & EDR & SARL & 2000 & & $\begin{array}{l}\text { C. Neuschwander } \\
\text { F. Ampe (D) }\end{array}$ \\
\hline \multirow[t]{2}{*}{ Cabinet Transversal } & Transversal & $\begin{array}{l}\text { Profession } \\
\text { libérale }\end{array}$ & 2003 & & J.-M. Roux \\
\hline & & $\begin{array}{l}\text { Profession } \\
\text { libérale }\end{array}$ & 2003 & & J.-Y. Chapuis (D) \\
\hline
\end{tabular}


Tableau 2 : Profils professionnels des consultants enquêtés

\begin{tabular}{|c|c|c|c|c|}
\hline Nom & Formation & $\begin{array}{l}\text { Activités de conseil } \\
\text { et d'études }\end{array}$ & $\begin{array}{l}\text { Activités } \\
\text { d'enseignement }\end{array}$ & $\begin{array}{l}\text { Mandats électifs / } \\
\text { Cabinets politiques }\end{array}$ \\
\hline $\begin{array}{l}\text { Francis } \\
\text { AMPE }\end{array}$ & $\begin{array}{l}\text { École Centrale } \\
\text { Paris } \\
\text { Économie } \\
\text { Psycho- } \\
\text { sociologie }\end{array}$ & $\begin{array}{l}\text { Urbanisme, stratégie } \\
\text { et aménagement du } \\
\text { territoire }\end{array}$ & ENPC & $\begin{array}{l}\text { Conseiller municipal / } \\
\text { VP intercommunal } \\
\text { Maire Chambéry } \\
\text { Conseiller régional }\end{array}$ \\
\hline Bernard ARCHER & $\begin{array}{l}\text { École Centrale } \\
\text { Paris } \\
\text { Doctorat } \\
\text { Urbanisme } \\
\text { IU Grenoble }\end{array}$ & $\begin{array}{l}\text { Planification urbaine } \\
\text { Études économiques } \\
\text { Recherche urbaine }\end{array}$ & IU Grenoble & $\begin{array}{l}\text { Directeur des } \\
\text { services techniques } \\
\text { (Grenoble) }\end{array}$ \\
\hline $\begin{array}{l}\text { Jean-Pierre } \\
\text { CHARBONNEAU }\end{array}$ & CNAM & $\begin{array}{l}\text { Politiques urbaines } \\
\text { et culturelles - } \\
\text { Urbanisme }\end{array}$ & & $\begin{array}{l}\text { Conseiller technique } \\
\text { pour les élus }\end{array}$ \\
\hline $\begin{array}{l}\text { Jean-Yves } \\
\text { CHAPUIS }\end{array}$ & $\begin{array}{l}\text { Droit } \\
\text { Sociologie } \\
\text { Urbanisme }\end{array}$ & $\begin{array}{l}\text { Stratégie urbaine et } \\
\text { projet urbain }\end{array}$ & $\begin{array}{l}\text { Directeur École } \\
\text { d'Architecture } \\
\text { IFU }\end{array}$ & $\begin{array}{l}\text { Conseiller municipal } \\
\text { /VP intercommunal } \\
\text { Rennes }\end{array}$ \\
\hline $\begin{array}{l}\text { Christophe } \\
\text { CIZERON }\end{array}$ & Sciences Po & $\begin{array}{l}\text { Développement } \\
\text { économique local - } \\
\text { Stratégie et Territoire }\end{array}$ & & $\begin{array}{l}\text { Directeur de cabinet } \\
\text { (Grand Lyon) }\end{array}$ \\
\hline $\begin{array}{l}\text { Jacques } \\
\text { De COURSON }\end{array}$ & $\begin{array}{l}\text { Doctorat } \\
\text { Économie } \\
\text { Urbanisme } \\
\text { IU Paris }\end{array}$ & $\begin{array}{l}\text { Prospective urbaine } \\
\text { et territoriale, } \\
\text { projets urbains, } \\
\text { développement local } \\
\text { et aménagement du } \\
\text { territoire }\end{array}$ & $\begin{array}{l}\text { ENPC } \\
\text { IU Paris } \\
\text { École Centrale } \\
\text { Paris }\end{array}$ & \\
\hline $\begin{array}{l}\text { Dominique } \\
\text { GAUDRON }\end{array}$ & $\begin{array}{l}\text { EHEC Paris } \\
\text { Doctorat } \\
\text { Économie }\end{array}$ & $\begin{array}{l}\text { Développement } \\
\text { économique local - } \\
\text { Stratégie et Territoire }\end{array}$ & & $\begin{array}{l}\text { Directeur de cabinet } \\
\text { (Chambéry) }\end{array}$ \\
\hline $\begin{array}{l}\text { Hervé } \\
\text { HUNTZINGER }\end{array}$ & $\begin{array}{l}\text { Doctorat } \\
\text { Économie }\end{array}$ & $\begin{array}{l}\text { Économie urbaine } \\
\text { et régionale - } \\
\text { Planification } \\
\text { territoriale }\end{array}$ & $\begin{array}{l}\text { École Nationale } \\
\text { des Travaux } \\
\text { Publics de l'État } \\
\text { IU Lyon }\end{array}$ & \\
\hline $\begin{array}{l}\text { Claude } \\
\text { NEUSCHWANDER }\end{array}$ & $\begin{array}{l}\text { École Centrale } \\
\text { Paris } \\
\text { Urbanisme } \\
\text { IU Paris }\end{array}$ & $\begin{array}{l}\text { Développement } \\
\text { économique } \\
\text { territorial, } \\
\text { communication, } \\
\text { Aménagement du } \\
\text { territoire }\end{array}$ & & \\
\hline $\begin{array}{l}\text { Rémy } \\
\text { PRUD'HOMME }\end{array}$ & $\begin{array}{l}\text { EHEC Paris } \\
\text { Sciences Po } \\
\text { Doctorat } \\
\text { Économie }\end{array}$ & $\begin{array}{l}\text { Développement } \\
\text { économique } \\
\text { économie urbaine, } \\
\text { transports urbains, } \\
\text { aménagement } \\
\text { du territoire, } \\
\text { environnement }\end{array}$ & $\begin{array}{l}\text { IU Paris } \\
\text { ENPC }\end{array}$ & \\
\hline $\begin{array}{l}\text { Jean-Michel } \\
\text { ROUX }\end{array}$ & $\begin{array}{l}\text { INAPG } \\
\text { Doctorat } \\
\text { Géographie } \\
\text { économique }\end{array}$ & $\begin{array}{l}\text { Urbanisme - } \\
\text { Économie, montage } \\
\text { et programmation } \\
\text { des projets urbains }\end{array}$ & CNAM & \\
\hline
\end{tabular}

IU : Institut d'Urbanisme / IFU : Institut Français d'Urbanisme / INAPG : Institut National Agronomique Paris-Grignon / ENPC : École Nationale des Ponts et Chaussées / EHEC : École des Hautes Etudes Commerciales / CNAM : Conservatoire National des Arts et Métiers 
Tableau 3 : Entretiens réalisés dans le cadre du programme CHRISTIANA - Axe 1

\begin{tabular}{|c|c|c|}
\hline Nom & Date(s)/Lieu & Éléments marquants du parcours professionnel \\
\hline $\begin{array}{l}\text { Jacques } \\
\text { de Courson } \\
*\end{array}$ & $\begin{array}{l}\text { 03/11/2009 } \\
\text { Paris }\end{array}$ & $\begin{array}{l}\text { Chargé d'études économiques Agence d'urbanisme de Lille (1974- } \\
\text { 1977), Directeur d'études Service STU Ministère de I'Urbanisme et } \\
\text { du Logement (1977-1984), Directeur adjoint Agence d'urbanisme } \\
\text { de Lyon (1984-1990), Consultant associé Ten / Algoé (1990-2005), } \\
\text { Professeur associé IUP, ENPC, ECP. Auteur de plusieurs ouvrages, } \\
\text { Président fondateur de l'association Urbanistes du monde depuis } \\
2005\end{array}$ \\
\hline $\begin{array}{l}\text { Jean Clément } \\
*\end{array}$ & $\begin{array}{l}\text { 01/12/2009 } \\
\text { Lyon }\end{array}$ & $\begin{array}{l}\text { Employé au service d'organisation scientifique du travail d'une } \\
\text { entreprise industrielle lyonnaise (1953-1960), Directeur technique } \\
\text { d'une entreprise industrielle grenobloise (1960-1962), Consultant, } \\
\text { Directeur général puis PDG Algoé et Président Syntec Conseil en } \\
\text { management (1963-1996). Trésorier E \& H (1996-2007), Membre } \\
\text { du Conseil de développement du Grand Lyon depuis } 2006\end{array}$ \\
\hline $\begin{array}{l}\text { Jean Frébault } \\
\text { * }\end{array}$ & $\begin{array}{l}01 / 12 / 2009 \\
22 / 02 / 2011 \\
\text { Lyon }\end{array}$ & $\begin{array}{l}\text { X - Ponts et Chaussées, Ministère de l'Équipement (1966- } \\
\text { 1970), Directeur Agence d'urbanisme de Toulouse (1971-1977), } \\
\text { Directeur Agence d'urbanisme de Lyon (1978-1988), Directeur } \\
\text { de l'Architecture et de I'Urbanisme du Ministère de l'Équipement } \\
\text { (1989-1993), Directeur EPIDA (1994-1999), Président 5' section } \\
\text { "Aménagement et environnement » du Conseil Général des } \\
\text { Ponts et Chaussées (1999-2005), Président du Conseil de } \\
\text { Développement du Grand Lyon depuis 2006, } \\
\text { Membre de l'association Urbanistes du monde depuis } 2005\end{array}$ \\
\hline $\begin{array}{l}\text { Jean-Pierre } \\
\text { Aldeguer } \\
*\end{array}$ & $\begin{array}{l}\text { 15/02/2010 } \\
\text { Lyon }\end{array}$ & $\begin{array}{l}\text { Chargé d'études CCI de Lyon (1970-1972), Chargé d'études GEP } 2 \\
\text { DDE du Rhône (1973-1975), Directeur adjoint Atelier puis Agence } \\
\text { d'urbanisme de Lyon (1975-1983), Directeur adjoint SERL (1984- } \\
\text { 1988), Directeur SED Haute-Savoie (1989-années 2000) }\end{array}$ \\
\hline François Bregnac & $\begin{array}{l}16 / 03 / 2010 \\
\text { Lyon }\end{array}$ & $\begin{array}{l}\text { Architecte urbaniste libéral (1979-1983), Chargé d'études, } \\
\text { Conseiller scientifique, Directeur et Directeur adjoint de l'Agence } \\
\text { d'urbanisme de Lyon depuis } 1983\end{array}$ \\
\hline $\begin{array}{l}\text { Dominique } \\
\text { Gaudron }\end{array}$ & $\begin{array}{l}\text { 22/03/2010 } \\
\text { Lyon }\end{array}$ & $\begin{array}{l}\text { Enseignant d'économie en lycée (1969-1974), Animateur } \\
\text { socioculturel (1975), Permanent Centre des Jeunes Agriculteurs de } \\
\text { Savoie (1976-1977), Directeur de cabinet du Maire de Chambéry } \\
\text { (1977-1983), Consultant associé en développement local Ten / } \\
\text { Algoé (1984-2010) } \\
\text { Membre de l'association Urbanistes du monde depuis } 2005\end{array}$ \\
\hline $\begin{array}{l}\text { Vincent Berthet } \\
*\end{array}$ & $\begin{array}{l}31 / 03 / 2010 \\
\text { Lyon }\end{array}$ & $\begin{array}{l}\text { Animateur socioculturel, Directeur de Centre Social et Journaliste } \\
\text { pigiste multi-titres Le Progrès, La Croix, Phosphore, Le Pèlerin } \\
\text { (1982-1988), Journaliste Radio Chrétienne de France (1988-1990), } \\
\text { Chargé d'études et animateur de la revue E \& H (1990-2007), } \\
\text { Consultant Développement \& Humanisme } \\
\text { Directeur de cabinet du maire de Saint-Étienne depuis } 2008\end{array}$ \\
\hline
\end{tabular}




\begin{tabular}{|c|c|c|}
\hline $\begin{array}{l}\text { Jean-Philippe } \\
\text { Motte }\end{array}$ & $\begin{array}{l}01 / 04 / 2010 \\
19 / 04 / 2010 \\
\text { Grenoble }\end{array}$ & $\begin{array}{l}\text { Chargé d'études OREAM Nord (1964-1969), Agence d'urbanisme } \\
\text { de Grenoble (1970-1983), CCAS Ville de Grenoble (1983-1985), } \\
\text { Animateur revue Économie \& Humanisme (1987-1990), Créateur } \\
\text { et délégué association INUDEL (1985-1995), Président CRDSU } \\
\text { Rhône-Alpes (2001-2008), } \\
\text { Élu à la Ville de Grenoble et à la Métro en charge de la Politique } \\
\text { de la Ville, du CCAS, des secteurs GUSP et de la démocratie } \\
\text { participative (1995-2008) }\end{array}$ \\
\hline Christian Dupré & $\begin{array}{l}\text { 07/05/2010 } \\
\text { Grenoble }\end{array}$ & $\begin{array}{l}\text { Chargé d'études SADI (1974-1989), Chargé d'études SCET (1989- } \\
\text { 2003), Chargé de mission Prospective, Stratégie et Aménagement } \\
\text { métropolitain Grenoble La Métro (2003-2010) }\end{array}$ \\
\hline Michel Rousselot & $\begin{array}{l}\text { 29/11/2010 } \\
\text { Paris }\end{array}$ & $\begin{array}{l}\text { X-Ponts et Chaussées, IGP, Directeur du Service des Affaires } \\
\text { Économiques et Internationales du Ministère des TP - Équipement } \\
\text { (SAEI) et animateur Division Transports (1960-1968), Chef du } \\
\text { Service Régional et Urbain du CGP (1969-1973), Directeur EPA Ville } \\
\text { Nouvelle Marne-la-Vallée (1974-1981), Directeur Cabinet Ministre } \\
\text { de l'Équipement (1981-1982; 1991-1992), Directeur du personnel } \\
\text { Ministère de l'Équipement (1982-1985), RATP (1985-1991), Directeur } \\
\text { Régional de l'Équipement lle-de-France (1992-années 2000) }\end{array}$ \\
\hline $\begin{array}{l}\text { Rémy } \\
\text { Prud'homme }\end{array}$ & $\begin{array}{l}\text { 29/11/2010 } \\
\text { Paris }\end{array}$ & $\begin{array}{l}\text { Professeur des universités en Sciences économiques depuis } \\
\text { 1966 et à I'IUP (1977-2007), Conseiller CERAU-BETURE (1969- } \\
\text { 1973), Directeur adjoint de l'Environnement OCDE (1974-1977), } \\
\text { Consultant et expert international depuis 1972, Fondateur et } \\
\text { directeur de L'CFIL (1980-1988) }\end{array}$ \\
\hline $\begin{array}{l}\text { Hervé } \\
\text { Huntzinger }\end{array}$ & $\begin{array}{l}30 / 11 / 2010 \\
\text { Paris }\end{array}$ & $\begin{array}{l}\text { Économiste Adjoint CERAU-BETURE (1968-1971), Chargé d'études } \\
\text { SACEC (1976-1979), membre du Groupe de thèmes urbains OCDE } \\
\text { (1980-1995), Consultant Économie urbaine et territoriale - Directeur } \\
\text { associé TETRA depuis 1980, Professeur associé CUD, IUL, ENTPE }\end{array}$ \\
\hline $\begin{array}{l}\text { Jean-Michel } \\
\text { Roux }\end{array}$ & $\begin{array}{l}30 / 11 / 2011 \\
\text { Paris }\end{array}$ & $\begin{array}{l}\text { Administrateur civil de banque Caisse nationale du Crédit } \\
\text { Agricole (1966-1970), Consultant Urbanisme, Développement } \\
\text { local et Aménagement du territoire AREA et CODRA (1970-1987), } \\
\text { Directeur de développement Ressources et Valorisation (R\&V) } \\
\text { Filiale aménagement urbain de la CGE (1987-1991), Directeur } \\
\text { de projets urbains SCET (1991-2003), Consultant en urbanisme } \\
\text { Transversal depuis 2003, Professeur associé au CNAM } \\
\text { Auteur de plusieurs ouvrages }\end{array}$ \\
\hline $\begin{array}{l}\text { Christophe } \\
\text { Cizeron }\end{array}$ & $\begin{array}{l}\text { 06/12/2011 } \\
\text { Lyon }\end{array}$ & $\begin{array}{l}\text { Responsable de développement dans une structure de promotion } \\
\text { immobilière spécialisée dans l'urbanisme commercial et } \\
\text { l'immobilier d'entreprises (1988-1993), Consultant en stratégie } \\
\text { auprès des collectivités locales Algoé (1994-2002), Conseiller } \\
\text { technique et Directeur de cabinet du Président du Grand Lyon } \\
\text { (2003-2009), Directeur du développement Gestion d'espaces GL } \\
\text { Events depuis } 2009\end{array}$ \\
\hline $\begin{array}{l}\text { Bernard Archer } \\
*\end{array}$ & $\begin{array}{l}\text { 03/01/2011 } \\
\text { Grenoble }\end{array}$ & $\begin{array}{l}\text { Chargé d'études SCET - études générales (1960-1963), Chargé } \\
\text { d'études et directeur SORETUR (1963-1967), Directeur d'études } \\
\text { BERU (1967-1973), Directeur des études économiques et des } \\
\text { programmes SCIC/CDC (1975-1977), Directeur des services } \\
\text { techniques Ville de Grenoble (1977-1984), Premier Secrétaire et } \\
\text { Directeur du Plan Urbain du Ministère de l'Équipement (1984-1987), } \\
\text { Directeur de l'Institut National du Génie urbain de Lyon (1987-1995) }\end{array}$ \\
\hline
\end{tabular}

Entretiens réalisés par $R$. Linossier, sauf ceux marqués par un *, qui ont été réalisés par $R$. Linossier et d'autres participants du programme CHRISTIANA (F. Belmessous, T. Ben Mabrouk, J.-P. Blais, O. Chatelan, L. Combe, C. Leclercq, M. Prévot) 